\title{
Les indicateurs de l'évolution actuelle des lits fluviaux. Etude de cours d'eau du Nord-Est de la
}

\section{France}

Indicators of the current evolution of river beds. Study on rivers in north-east

France

Jeannine Corbonnois, Thomas Jacquemot, Loris Giovannacci, Thierry Beck et Mohamed El Ghachi

\section{OpenEdition \\ Journals}

Édition électronique

URL : http://journals.openedition.org/rge/3427

DOI : $10.4000 /$ rge.3427

ISSN : 2108-6478

Éditeur

Association des géographes de l'Est

Édition imprimée

Date de publication : 23 août 2011

ISSN : 0035-3213

\section{Référence électronique}

Jeannine Corbonnois, Thomas Jacquemot, Loris Giovannacci, Thierry Beck et Mohamed El Ghachi, "Les indicateurs de l'évolution actuelle des lits fluviaux. Etude de cours d'eau du Nord-Est de la France », Revue Géographique de l'Est [En ligne], vol. 51 / 3-4 | 2011, mis en ligne le 28 août 2012. consulté le 08 septembre 2020. URL : http://journals.openedition.org/rge/3427 ; DOI : https://doi.org/ 10.4000/rge.3427

Ce document a été généré automatiquement le 8 septembre 2020

Tous droits réservés 


\section{Les indicateurs de l'évolution actuelle des lits fluviaux. Etude de cours d'eau du Nord-Est de la France}

Indicators of the current evolution of river beds. Study on rivers in north-east

France

Jeannine Corbonnois, Thomas Jacquemot, Loris Giovannacci, Thierry

Beck et Mohamed El Ghachi

\section{Introduction}

Les travaux effectués depuis plusieurs années par le CEGUM pour la DIREN Lorraine et l'Agence de l'Eau Rhin-Meuse ont porté sur la dynamique des cours d'eau du nord-est de la France. Ils ont permis de rassembler de nombreuses données dans le but de caractériser les lits fluviaux. En sont extraits une partie des éléments utilisés pour mener cette réflexion. Sur ces cours d'eau, les tronçons les plus dynamiques servent à repérer les indicateurs de la morphogenèse. Description, localisation et fréquence d'apparition le long des rivières, associées à l'analyse des modifications morphologiques récentes (quelques décennies) permettent de déterminer les conditions de l'évolution actuelle des lits. Les résultats obtenus sont révélateurs de modes de fonctionnement qui à l'exception de celui de la Moselle, étaient peu étudiés jusqu'à présent. Ils peuvent servir d'aide à la gestion des rivières et des fonds alluviaux en général.

2 La mise en évidence de la dynamique des lits fluviaux est faite en deux étapes :

- présentation générale du cadre régional et des traitements statistiques effectués ;

- mise en évidence des indicateurs simples ou combinés de l'évolution actuelle à partir de

l'interprétation de tous les résultats, et de la connaissance du terrain. 


\section{Physionomie des lits fluviaux}

\section{A. Les conditions régionales}

3 Le nord-est de la France est parcouru par un réseau hydrographique dense. A l'exception de la Moselle, il comprend des rivières (Fig. 1), longues de moins de 200 km, qui drainent des bassins versants de 500 et $3000 \mathrm{~km}^{2}$ de superficie. Les caractéristiques hypsométriques, géomorphologiques et climatiques de l'espace régional leur assurent une bonne alimentation en eau ; nombreux sont les cours d'eau qui se forment dans les Vosges. Leur fonctionnement est commandé par un contexte « montagnard » amont où l'eau est collectée. Il cède rapidement la place à des plateaux situés en contrebas par lesquels cette eau transite. Du côté lorrain des Vosges qui seul nous intéresse ici, c'est là que se manifeste la dynamique fluviale. Elle comprend le plus souvent une redistribution de matériaux hérités, prélevés dans les berges ou au fond des lits fluviaux, se réalisant sous l'influence d'actions anthropiques.

Figure 1 : Carte de repérage

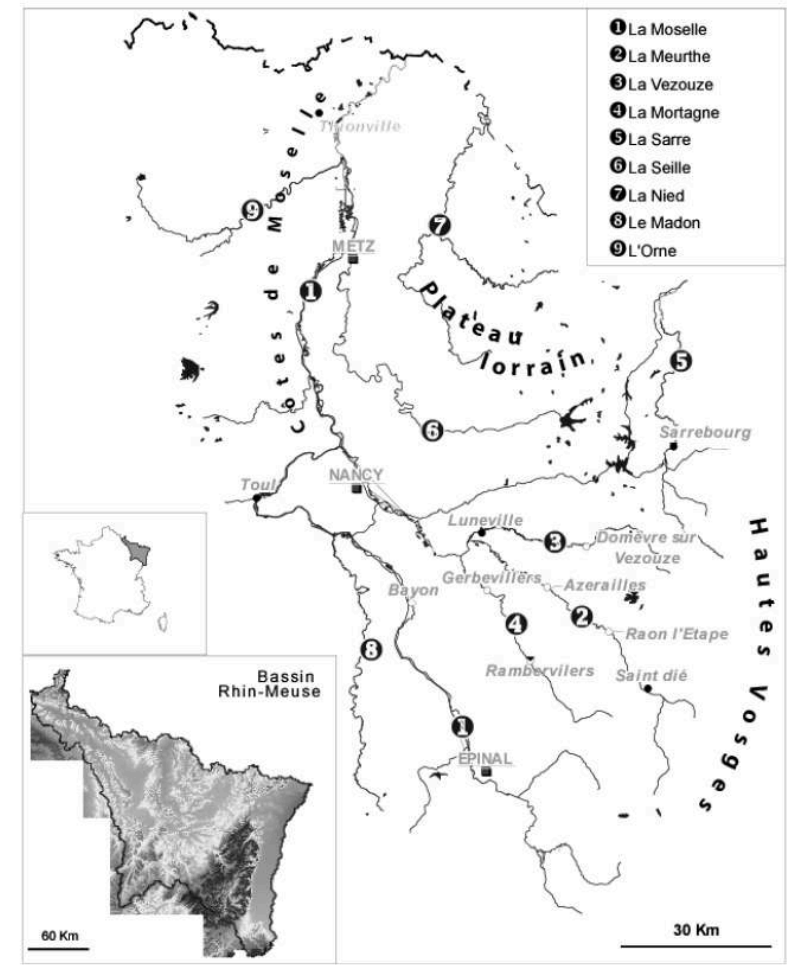

\section{La dimension des lits fluviaux}

Les cours d'eau parcourent des vallées profondes de 300 à $600 \mathrm{~m}$ dans les Vosges et de moins de $300 \mathrm{~m}$ en Lorraine. Leur largeur est influencée par les conditions structurales du substratum encaissant (résistance des roches et tectonique), guidant la dynamique fluviale en favorisant la dilatation ou la contraction des fonds alluviaux. Ceux-ci, inclinés de quelques pourcents en tête de bassin, présentent vers l'aval des pentes faibles comprises entre 0,6 et $1 \%$ o. Ils sont remblayés de matériaux épais de 3 à $7 \mathrm{~m}$, alluvions grossières à la base surmontées sur $2 \mathrm{~m}$ au maximum par des limons sableux. 
Les rivières, bien alimentées en raison de précipitations régulières et abondantes (environ $800 \mathrm{~mm}$ en Lorraine et plus de $1100 \mathrm{~mm}$ sur les Vosges), écoulent des débits de 8 à $12 \mathrm{l} / \mathrm{s} / \mathrm{km}^{2}$ qui augmentent à l'approche du massif montagneux pour atteindre 18, voire plus de $22 \mathrm{l} / \mathrm{s} / \mathrm{km}^{2}$ (Corbonnois, Humbert, 2000). Au cours de l'année, leur régime est pluvial océanique. Il n'y a pas d'influence nivale notoire. Les extrêmes hydrologiques, comprennent des étiages survenant en août ou septembre (1,5 à 3,5 l/s/ $\mathrm{km}^{2}$ en débit mensuel de fréquence $1 / 5$, AERM 1996) et des crues intervenant le plus souvent pendant la période hivernale (de l'ordre de $250 \mathrm{l} / \mathrm{s} / \mathrm{km}^{2}$ en débit moyen journalier pour une fréquence $1 / 10$ ). Aucun événement d'importance majeure n'est survenu ces dernières années ; ainsi nos observations de terrain effectuées entre 1999 et 2005 portent-elles des marques « banales » de l'évolution géomorphologique.

5 Comme ailleurs, les fonds alluviaux du nord-est de la France ont été modifiés par les actions anthropiques. Barrage, fixation de berges, curage, rectification du tracé concernent le lit mineur, voies de communications, constructions diverses, mise en valeur agricole, exploitation d'alluvions, etc., concernent le lit majeur. Ces modifications déterminent de la part des cours d'eau une réponse plus insidieuse que spectaculaire. Cela montre plutôt une bonne robustesse à l'impact de facteurs externes, anthropiques et également hydrologiques. Seules les plus fortes crises provoquent des ajustements morphologiques, d'ailleurs lents à se répercuter.

\section{Les formes fluviales}

6 Elles ont été identifiées à partir de relevés de terrain, complétés par l'interprétation de divers documents (anciennes cartes, photographies aériennes IGN et à basse altitude). Les données ont été cartographiées au 1/10000ème ou au 1/5000ème selon la dimension des organismes fluviaux (CEGUM/DIREN, 2001, 2002, 2003). Les éléments pris en considération sont :

- pour le lit mineur, le tracé du chenal unique (Fig.2) comprenant des sinuosités libres ou ancrées très variées, (boucles, méandres réguliers ou en oméga formant alors des cornes), l'allure des sections mouillées à pleins bords (largeur du chenal et hauteur des berges) et l'état des berges (inclinaison, marques d'érosion, type de végétation, protection artificielle); - pour le lit majeur, les microformes (chenal de crue, ravine ou ancien lit mineur), la largeur et l'occupation du sol (Fig.3). 
Figure 2. Exemples de tracés (exemple de la Meurthe à Azerailles)

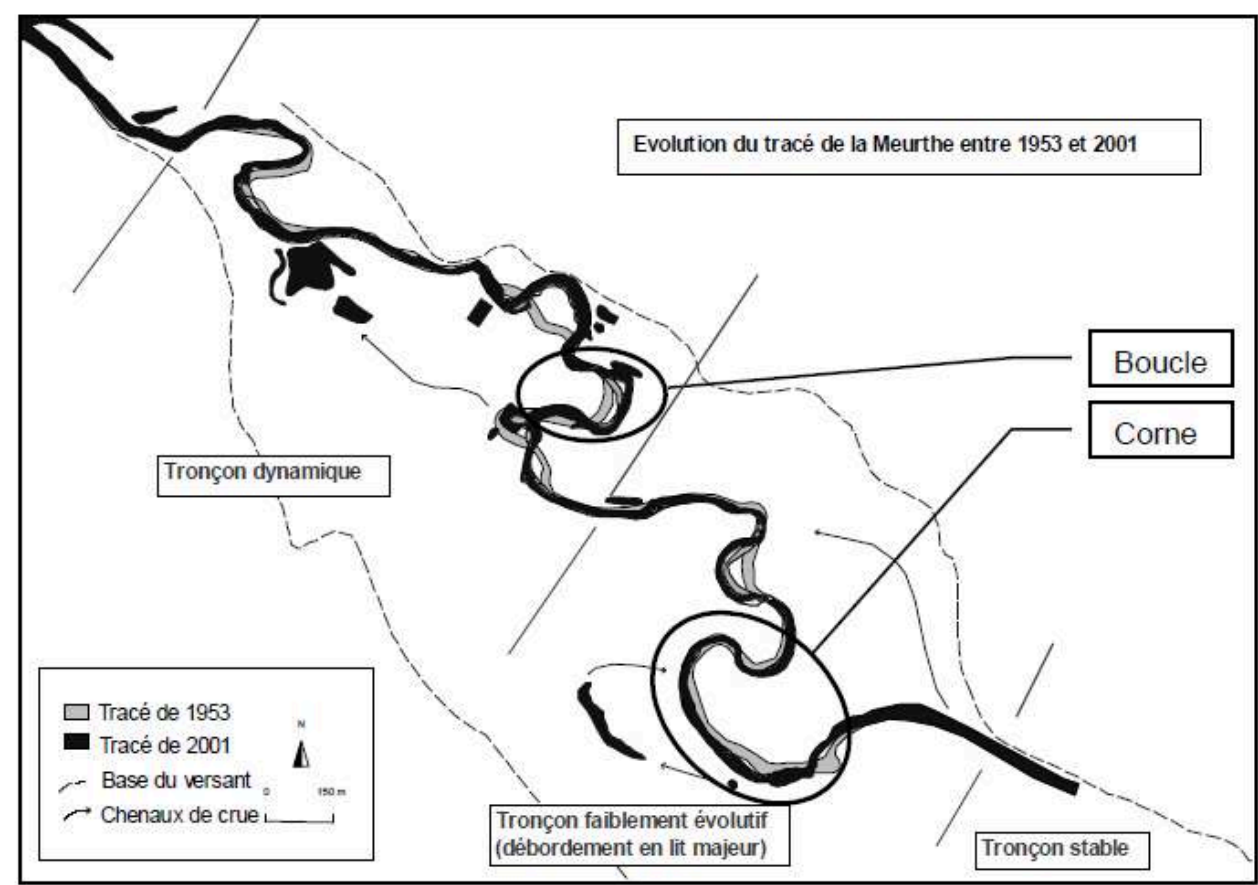

Figure 3 : Lit majeur d'une rivière. Exemple de la Meurthe à Azerailles

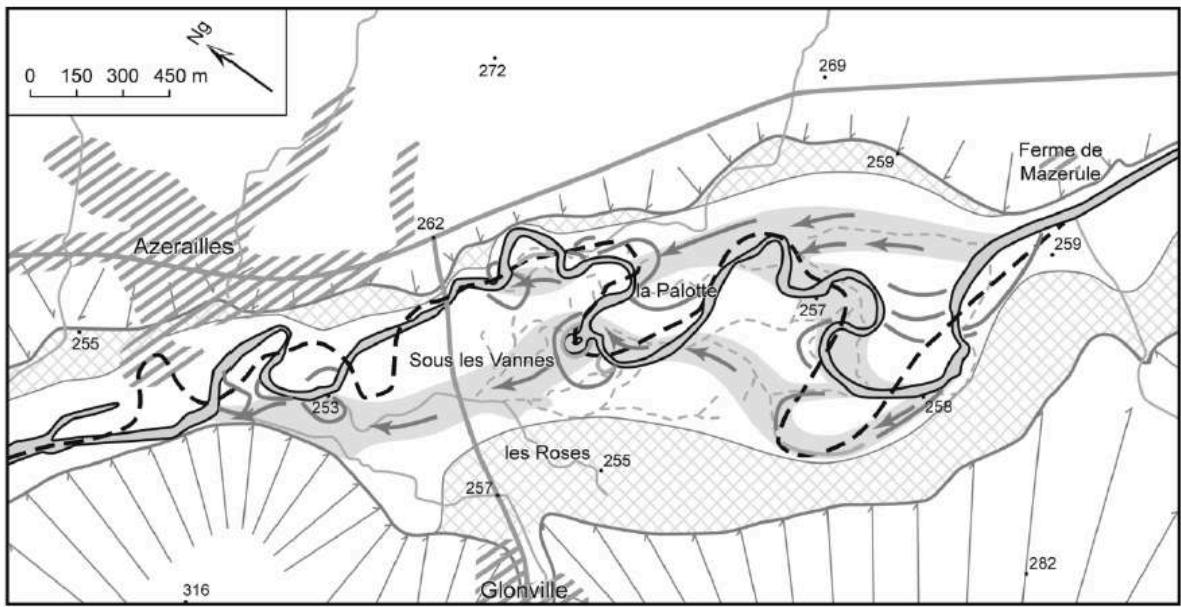

Morphologies des lits de la Meurthe - Secteur d'Azerailles

Passages en lit majeur :

Ancien bras (héritage)

Ancien bras fonctionnel au milieu du $X X^{e}$ siècle

Ruisseau

Chenal de crue / ravine

$\longrightarrow$ Flux préférentiels en crue (d'après les photos obliques en 1988, 1999 et 2002 et les observations de terrain)

Lit de débordement

\section{Tracés du lit mineur : Repères :} $\widetilde{E}$ En 1994
En 1822 (d'après
le cadastre)

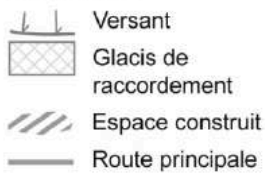

7 Ces formes fluviales présentent de nombreuses similitudes d'une rivière à l'autre. A l'exception de la Moselle, elles évoluent lentement sur une à plusieurs décennies ce qui 
allonge le temps d'adaptation des cours d'eau aux crises. Ainsi toutes les rivières présentent-elles des états de stabilité plus ou moins altérés.

- la Moselle en aval d'Epinal est la rivière la plus réactive. La destabilisation par les extractions de granulats en lit mineur arrêtées à la fin des années 70, a augmenté sa vulnérabilité aux crises. Elle présente de ce fait des formes fluviales vives assez spectaculaires et une déconnexion des lits mineurs et majeurs en raison d'une incision de la rivière de près de $2 \mathrm{~m}$ (Beck, 2003) ;

- la Meurthe moins concernée par l'extraction de granulats, ne présente une activité géomorphologique que sur certains secteurs. L'ajustement du cours d'eau à d'anciennes extractions se réalise sur plusieurs décennies, ce qui permet d'en suivre les états successifs (Corbonnois et al.,2003) ;

- la Vezouze et la Mortagne présentent des dynamiques fluviales notoires en raison de rectifications de tracés ou de fixations de berges;

- la Sarre, aménagée depuis longtemps (ancien canal, voie ferrée), n'a pas subi de modifications récentes. Les morphologies peu dynamiques sont stabilisées ; arbres et buissons occupent les berges ;

- enfin d'autres cours d'eau, qui drainent exclusivement le plateau lorrain comme le Madon ou la Seille ont une dynamique fluviale discrète, contenue par une pente longitudinale faible et des débits moins contrastés que ceux des rivières du pourtour vosgien.

Il est peu original de rappeler que les facteurs qui agissent sur la forme des lits fluviaux sont nombreux ; les conditions hydrologiques, hydrauliques et anthropiques commandent leur dynamique sous l'influence des dimensions longitudinales (pente en particulier), transversales (largeur des fonds alluviaux) et verticales des fonds alluviaux (Amoros et Petts, 1993). Ainsi se construit un schéma général de fonctionnement, valable à l'échelle régionale. A l'échelle locale, il comprend des variantes selon la caractéristique des rivières et les parties de cours d'eau concernées. Il peut être durablement modifié par les crises (crue anormalement forte ou aménagement spécifique), qui constituent des moments et des faits d'exception.

\section{B. Le traitement statistique des données, exemple de la Vezouze et de la Mortagne}

9 Pour ces deux cours d'eau, les formes fluviales ont été quantifiées par catégories et par longueurs de berge, à l'intérieur de tronçons homogènes (1000 à $2000 \mathrm{~m}$ de longueur), délimités sur le terrain. Les données ont ensuite fait l'objet de traitements multivariés (recherche de corrélations multiples et classification hiérarchique) pour déterminer leur poids respectif et les principales associations caractéristiques qui commandent l'état et la dynamique des lits (Jacquemot, 2004). Ainsi peut-on définir les indicateurs des formes stables et instables et leurs composants (variables utilisées pour le traitement statistique, Tableau 1) associés aux conditions régionales ou locales du milieu (Fig. 4, conditions principales et secondaires). Ces composants expliquent les raisons de la stabilité, (pente et fixation des berges d'origine naturelle ou anthropique) et de l'instabilité (hauteur des berges et alluvionnement dans le lit). Plus en détail, les corrélations révèlent d'autres liaisons intervenant de manière plus accessoire et ponctuelle. Ces résultats, qui tiennent compte des connexions lit mineur/lit majeur ont servi à représenter de façon synthétique les lits de la Mortagne et de la Vezouze par 
grands secteurs d'activité morphogène (Jacquemot, 2004). Ils servent également de modèle pour déterminer l'état des lits fluviaux sur les autres cours d'eau.

Tableau 1 : Liste des variables utilisées pour l'étude des corrélations

\begin{tabular}{|c|c|c|c|}
\hline & Variable & Code & Sources \\
\hline \multirow{9}{*}{ 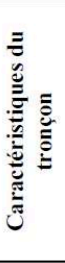 } & Altitude moyenne & Alti & AERM \\
\hline & Précipitation & Precip & AERM \\
\hline & Largeur en eau & LagE & SEQ-Eau \\
\hline & Largeur pleins-bords & LagP & SEQ-Eau \\
\hline & Pente & Pent & SEQ-Eau \\
\hline & Coefficient de sinuosité & $\mathrm{CoSi}$ & SEQ-Eau \\
\hline & Amplitude des méandres & Ampl & BdOrtho \\
\hline & Longueur d'onde & LOnd & BdOrtho \\
\hline & Rayon de courbure & Curv & BdOrtho \\
\hline \multirow{9}{*}{ 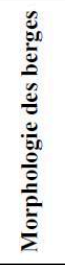 } & Hauteur moyenne de berge & $\mathrm{HtBg}$ & Terrain \\
\hline & Berge stable & Bges & Terrain \\
\hline & Berge dégradée & BgeD & Terrain \\
\hline & Berge sapée & $\mathrm{BgSp}$ & Terrain \\
\hline & Banquette & Banq & Terrain \\
\hline & Encoche & Enco & Terrain \\
\hline & Affaissement & Affa & Terrain \\
\hline & Ripisylve & Ripi & Terrain \\
\hline & Substratum dans les berges & SubB & Terrain \\
\hline \multirow{6}{*}{ 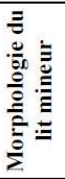 } & Banc vif & $\mathrm{BcVi}$ & Terrain \\
\hline & Banc végétalisé & $\mathrm{BcVe}$ & Terrain \\
\hline & Seuil naturel & SeiN & Terrain \\
\hline & Seuil construit & SeiC & Terrain \\
\hline & Embâcle & Emba & Terrain \\
\hline & Substratum dans le lit mineur & SubL & Terrain \\
\hline \multirow{8}{*}{ 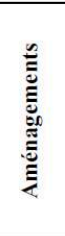 } & Enrochement & Enro & Terrain \\
\hline & Perre maçonné & Perr & Terrain \\
\hline & Digue & Digu & Terrain \\
\hline & Protection végétale & ProV & Terrain \\
\hline & Barrage & Barr & Terrain \\
\hline & Gravière & Grav & Terrain \\
\hline & Dérivation & Deri & Terrain \\
\hline & Recoupement de méandre & Reco & Terrain \\
\hline
\end{tabular}

Figure 4 : Résultats du traitement statistique

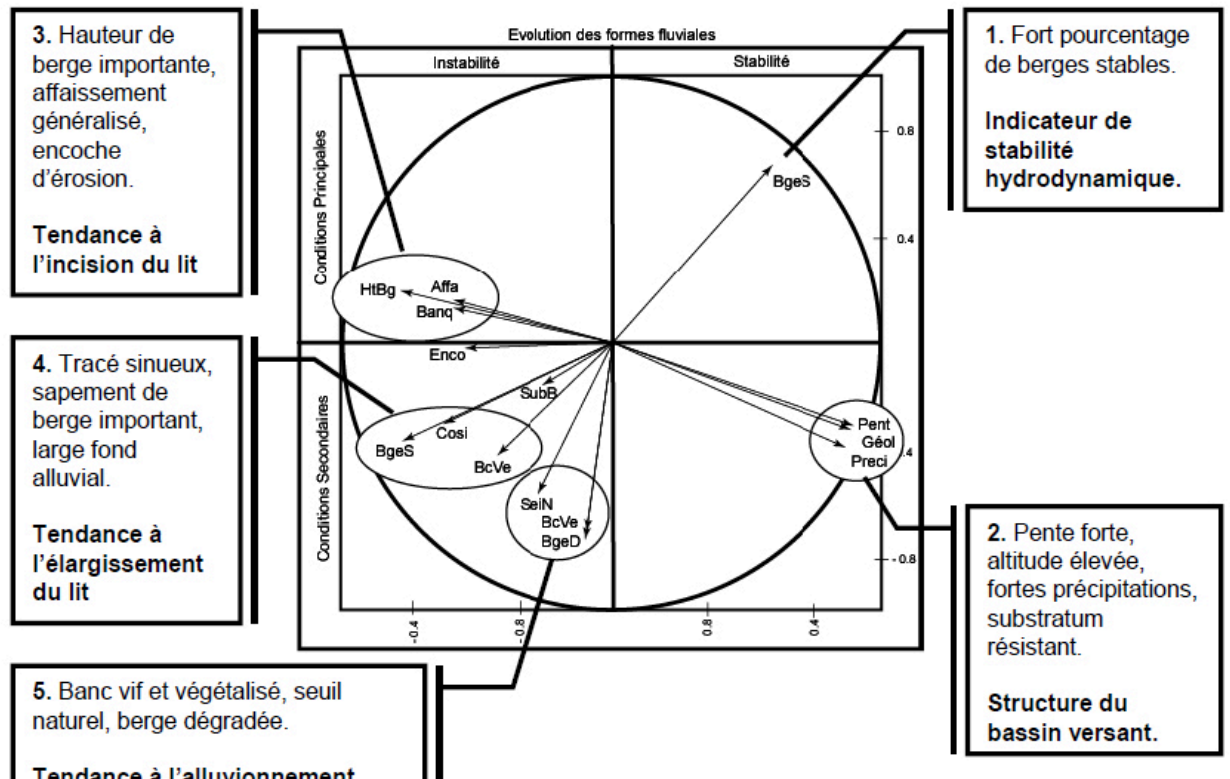




\section{Les indicateurs de l'évolution des lits}

Les lits fluviaux présentent généralement une résistance au changement (déterminé par les crues ou les actions anthropiques) plus ou moins grande. Les lits stables ou peu actifs et des lits instables, évolutifs ou mobiles que l'on peut ainsi définir, occupent des tronçons de cours d'eau de longueurs variées. Leur évolution est illustrée par la présentation d'un tronçon de Vezouze et de Meurthe.

\section{A. Assemblage des formes fluviales le long des cours d'eau}

Lits stables et instables sont assemblés de diverses manières de l'amont vers l'aval selon la caractéristique des fonds alluviaux liée aux conditions régionales et locales.

Dans le nord-est le la France, les lits stables sont le produit des conditions régionales, climat, caractéristiques des bassins versants (altitude, substratum), actions anthropiques.., qui construisent le schéma général de fonctionnement. L'équilibre dynamique relatif est défini par un lit mineur à berges basses (hauteur inférieure à 1,5 $m$ en période de basses eaux), souvent arborées. Le tracé inchangé depuis plusieurs décennies, voire siècles (Fig. 2), présente des sinuosités diverses, en lacets comme sur la Meurthe et la Mortagne, en cornes ou en méandres réguliers. Ces formes sont souvent associées à des débordements fréquents en lit majeur où les flux s'étalent, empruntent de larges chenaux de crue ou encore de courtes ravines de vidange (Fig. 3) ; ainsi correspondent-elles également à des tronçons de rivière où lit mineur et lit majeur sont en connexion. Cette stabilité peut être considérée comme un état " naturel », préservé des actions anthropiques récentes, les plus anciennes ayant pu être "digérées " par l'hydrosystème ; c'est notamment le cas de la Sarre dans sa traversée du plateau lorrain.

13 Comme l'ont montré les résultats du traitement statistique et leur cartographie (Jacquemot, 2004), les lits stables qui concernent en général plus de $60 \%$ du linéaire fluvial (à l'exception de la Moselle) sont situés sur différentes parties de cours d'eau (Fig. 5). En tête de bassin, ils présentent le plus souvent un tracé rectiligne (écoulement turbulent associé aux pentes fortes), des berges basses, stables (pavage par des matériaux grossiers, blocs hérités, apportés ou bien livrés par le substratum encaissant) et végétalisées (couvert forestier). Participent à la stabilité, des aménagements anciens, protections de berges fixant les chenaux ou anciennes canalisations guidant l'eau vers des moulins, détruits depuis. Vers l'aval, où la pente longitudinale est très faible (inférieure à $1 \%$ ), les lits stables moins fréquents, présentent un tracé sinueux (absorption de l'énergie fluviale disponible) et des berges basses qui favorisent des débordements précoces et fréquents (dispersion de l'énergie fluviale en lit majeur). Les actions anthropiques ont exploité et renforcé cette stabilité par des interventions variées (création de barrages et de dérivations, aménagements en vue de l'utilisation du lit majeur). 
Figure 5 : Liaisons entre pente et fréquence des berges sapées
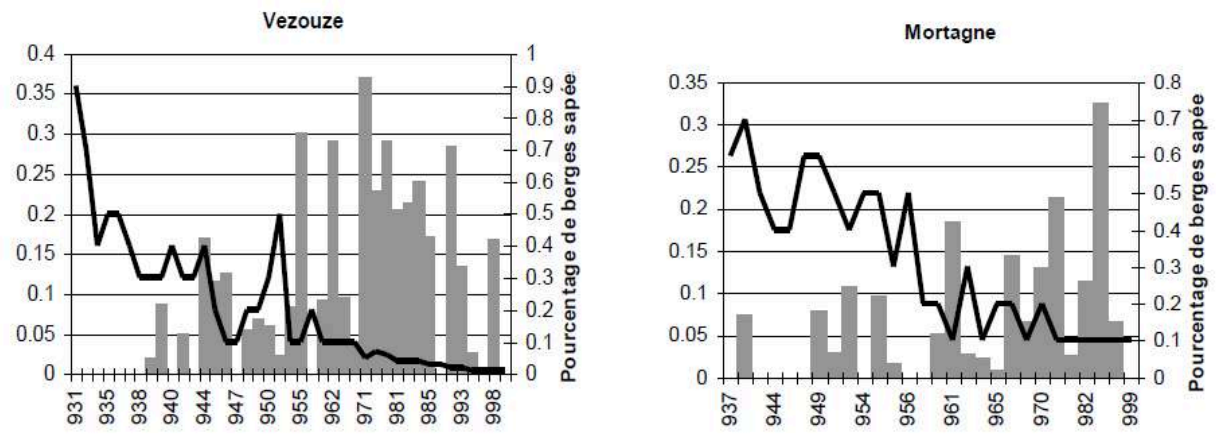

Les lits instables sont associés à des conditions locales (naturelles ou d'origine anthropique) qui altèrent les formes stables. Le lit mineur a des berges plus hautes (supérieures à $1,5 \mathrm{~m}$ pouvant atteindre jusqu'à $4 \mathrm{~m}$ ), à vif en raison de sapements liés directement à l'action fluviale ou combinés au glissement gravitaire de matériaux peu cohérents. Le tracé se modifie en quelques années. Il comprend des boucles se développant à partir d'encoches d'érosion ; elles s'étendent rapidement de quelques mètres à plusieurs dizaines de mètres. Cette évolution s'auto entretient ensuite. Fourniture de sédiments, formation de bancs et déviation du courant incitent au final à l'élargissement et au déplacement latéral du lit. Mais dans d'autres cas, les berges sont trop hautes et le débordement, retardé en période de crue, favorise l'incision du cours d'eau et la fixation du tracé. Le lit majeur, moins fréquemment sollicité, présente une topographie contrastée entre les anciens bras remblayés et les chenaux écoulant l'eau des crues débordantes les plus puissantes ; certains sont aménagés pour guider ces flux et limiter l'érosion des éléments naturels et des constructions.

Si les lits instables sont souvent dus aux interventions anthropiques, sur certains tronçons de rivières les conditions naturelles, irrégularité de la pente et de la largeur des fonds alluviaux, favorisent la fragilité ou augmentent la vulnérabilité. C'est le cas de la Moselle et de la Meurthe qui parcourent à l'amont d'anciennes vallées glaciaires comprenant une succession de verrous et d'ombilics. Ailleurs, les irrégularités sont liées aux facteurs structuraux (résistance des roches et tectonique). L'instabilité résulte de la construction dans les tronçons élargis ou très faiblement inclinés, d'un remblaiement alluvial sur lequel les rivières se déplacent : formation de plusieurs bras ou fréquents changements du tracé très sinueux (Fig.3). Mais des aménagements ont exploités les réalisations naturelles, les ont contraintes ou faites disparaître. Ainsi, constate-t-on que la quasi-totalité de la dynamique actuelle des lits fluviaux est expliquée par les interventions anthropiques variées, nombreuses et fréquentes : recoupement de sinuosités, modification de la courbure des méandres, fixation de berges, chenalisation entre les éléments construits en lit majeur ou encore modification des sections mouillées par le curage ou l'extraction de sédiments en lit mineur.... En général, l'instabilité apparue ponctuellement se propage vers l'amont ou vers l'aval et finit par affecter de longs tronçons de cours d'eau.

Mais les organismes fluviaux se différentient les uns des autres par le temps de réaction des cours d'eau, nécessaire pour l'ajustement aux nouvelles conditions. On peut compter ce temps (modification visible des morphologies et mesurables) en plusieurs dizaines d'années à l'exception de la Moselle qui réagit plus vite. 


\section{B. Evolution diachronique des secteurs actifs}

17 Dans les secteurs actifs, l'évolution des lits fluviaux se manifeste par des actions simples ou complexes selon les processus qui les déterminent. Variables selon les sites et les situations hydrologiques, ils façonnent des morphologies qui se juxtaposent ou se substituent les unes aux autres. Les morphologies les plus spectaculaires sont situées sur la Moselle et font l'objet des travaux de T. Beck (thèse en cours). Sur les autres cours d'eau, cette évolution est conduite par un mode dominant de réaction ; élargissement ou enfoncement du lit, intervenant plus ou moins rapidement après une crise, selon l'abondance et la nature des sédiments livrés par les berges et le fond du lit, la dimension du lit fluvial et la puissance des écoulements. Se crée ainsi toute une palette de formes vives et évolutives. Les tronçons de Vezouze et de Meurthe en donnent des illustrations.

\section{L'ajustement lent du lit de la Vezouze}

18 La rivière descend du Donon et rejoint la Meurthe à Lunéville (Fig.1). Dès le pied du versant lorrain des Vosges, elle arrive dans une large vallée $(2000 \mathrm{~m})$ établie dans les argiles du Keuper. Là, l'énergie acquise à l'amont et liée à une bonne alimentation pluviale, sert en grande partie à réaliser un tracé très sinueux. Dans le cadre d'aménagements ruraux, les boucles majeures ont été recoupées en 1988 (Fig. 6). Depuis, la Vezouze s'adapte lentement au nouveau tracé selon des modes complexes menant à l'enfoncement (de 50 à $80 \mathrm{~cm}$ ) ou à l'élargissement du lit. A titre d'exemples (Fig.7), les profils V1 et V3 montrent un élargissement, influencé par les travaux de calibrage mais également dû à un pavage d'alluvions grossières héritées, révélées par les travaux et gênant le creusement actuel. Ce creusement se cantonne aux endroits les plus sévèrement raccourcis comme en $\mathrm{V} 4$ (réduction de $2 / 3$ de la longueur du lit). A l'inverse, en V7 et V9 le lit a gardé sa forme construite lors des travaux de 1988.

19 Sur le terrain, d'autres dégradations sont visibles; glissement des matériaux sur les berges trop hautes et souvent verticales, formation de bancs et de banquettes.... Mais généralement, les ajustements sont discrets et l'inertie du système est sous le contrôle de la pente très faible.

20 Ce fonctionnement sur plusieurs décennies, est assez représentatif de la plupart des rivières qui parcourent le plateau lorrain, Seille (El Ghachi, 2004), Madon, Nied ou encore Meurthe aval (CEGUM/DIREN, 2002). 
Figure 6 : Les sinuosités sur la Vezouze à l'amont de Lunéville

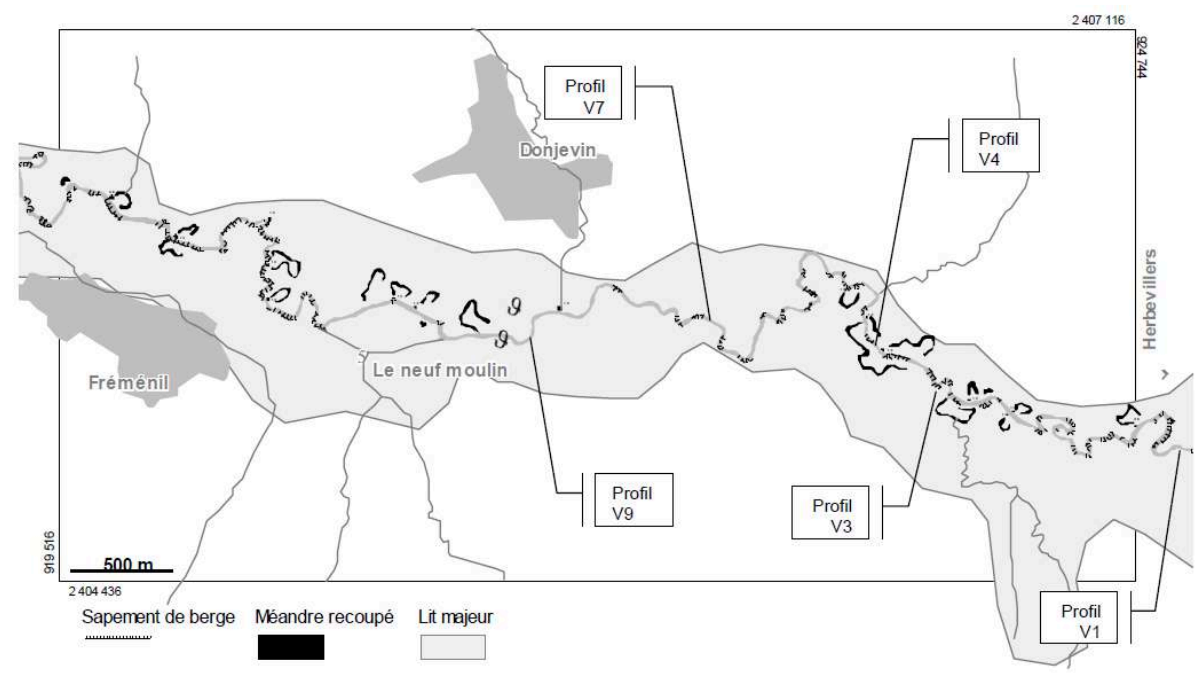

Figure 7 : Evolution du lit de la Vezouze après le recoupement de sinuosités

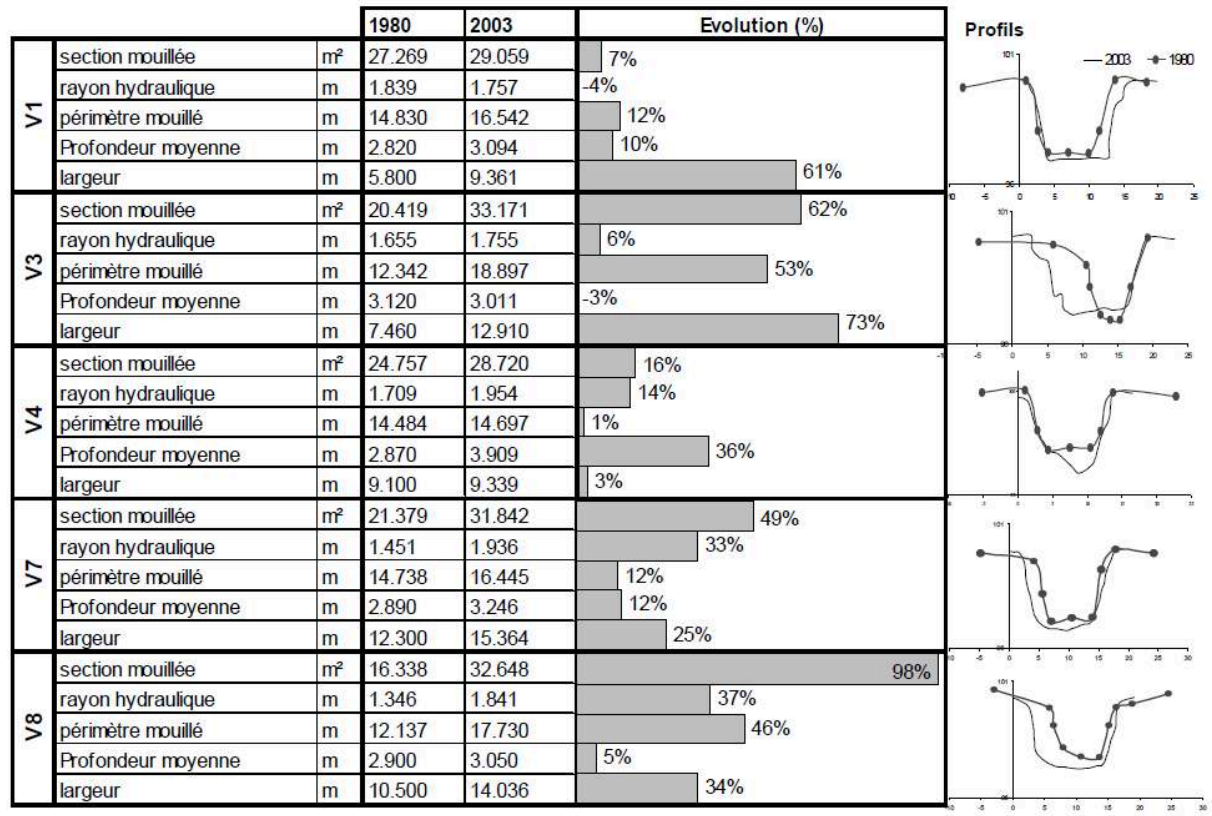

\section{L'évolution du lit de la Meurthe à Saint Michel (méandres de la Voivre)}

21 Ce tronçon, situé vers la sortie du massif vosgien (juste en aval de Saint Dié, Fig.1) doit ses caractéristiques à :

- des conditions régionales, pente marquée comprise entre 1,5 et $2 \%$ (Fig.8), resserrement de la vallée en aval du bassin Permien avec re- concentration des flux d'eau allégés de leur charge solide grossière en grande partie abandonnée en amont ;

- et des conditions locales, léger élargissement de la vallée entre deux secteurs plus étroits, aval de la partie canalisée dans la traversée de l'agglomération de Saint Dié, modification du lit par les extractions de granulats, en lit mineur jusqu'à la fin des années 70, puis en lit majeur. 
Il en résulte une activité morphogène, plus grande et des réactions plus rapides à tout changement $\mathrm{du}$ milieu. Les manifestations essentielles sont représentées par la modification du tracé intervenue depuis près d'un siècle (Fig.9) et une évolution notoire des sinuosités au cours des trois dernières décennies (Fig.10); exhaussement de tout le tronçon et recoupement de sinuosités par défluviation. Les modifications sont influencées par les actions anthropiques variées, aménagement pour la flottaison du bois, alimentation de moulins et plus récemment, extraction d'alluvions.

Ce schéma de fonctionnement se reproduit pour d'autres secteurs actifs, situés sur la Meurthe, plus en aval comme à Saint Clément, sur la Mortagne en aval de Rambervillers et sur la Moselle en aval d'Epinal.... La modification patente des morphologies fluviales est inscrite en particulier dans l'absence de végétation sur les berges ou sur les bancs d'alluvions qui encombrent le lit.

4 Mais ce schéma est affecté de variantes, selon le calibre des rivières, réglé par le débit, la charge solide et la pente des lits.

Figure 8 : Deux profils longitudinaux de la Meurthe entre Saint Dié et Raon l'Etape

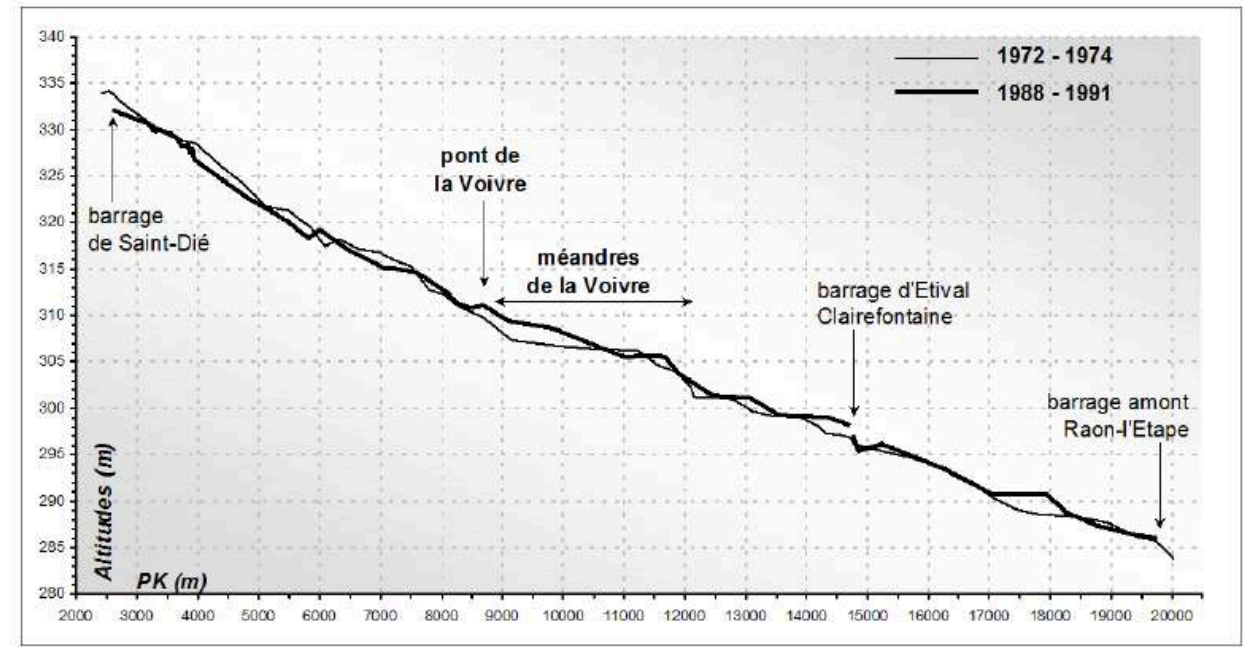

Figure 9 : Modification du tracé de la Meurthe au cours du 20ème siècle

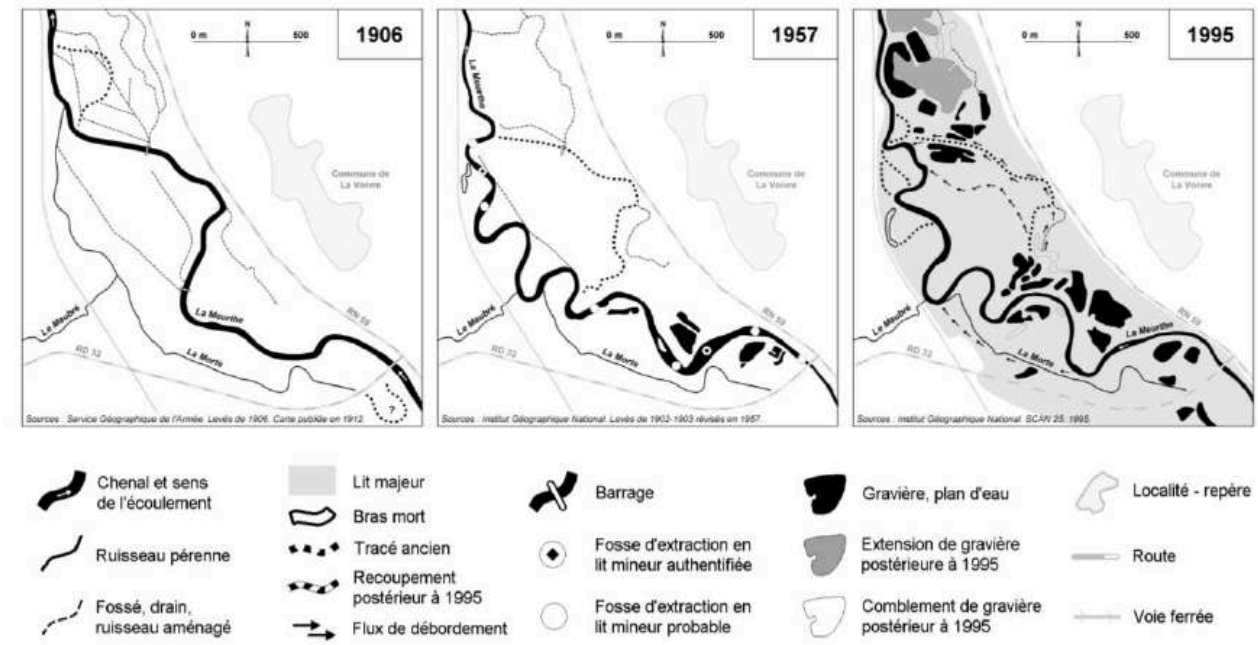




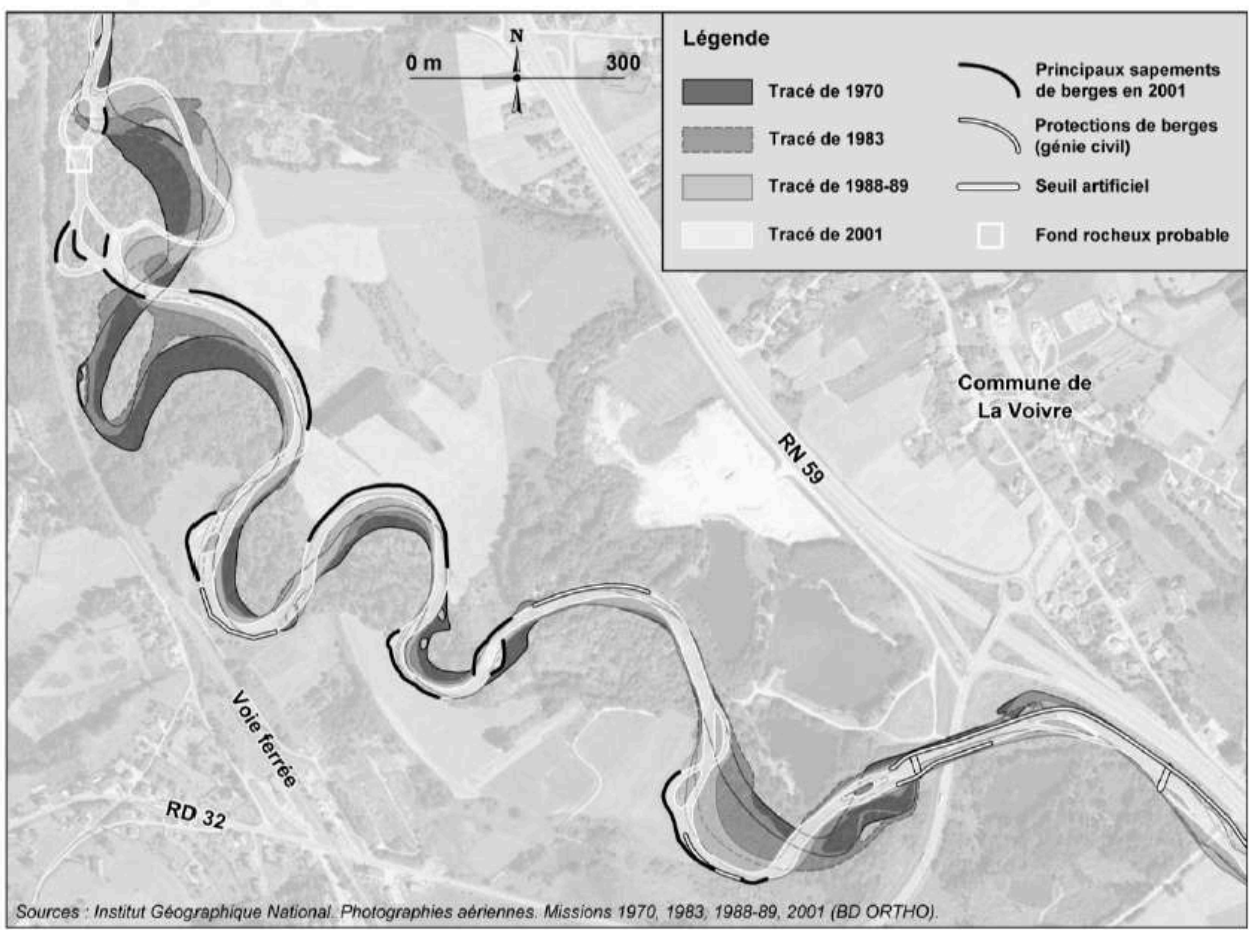

Les indicateurs de l'évolution actuelle des lits fluviaux d'abord liés aux conditions régionales et locales sont finalement résumés dans la forme des lits et des berges et la vitesse de leur modification. Parmi ces critères, la hauteur des berges joue un rôle important, elle commande les connexions rares ou fréquentes avec le lit majeur et agit, sous l'influence de la pente, sur la pérennité et la stabilité des formes dans l'ensemble $\mathrm{du}$ fond alluvial. La vitesse de cette évolution est très variable. On peut noter qu'entre la Vezouze et la Meurthe, elle varie du simple au double : d'importantes modifications sont intervenues sur la Meurthe en une dizaine d'années, des modifications plus ténues sont perceptibles sur la Vezouze vingt ans après des interventions drastiques... Cette vitesse est encore plus grande sur la Moselle.

\section{Conclusion}

Les lits fluviaux des cours d'eau du nord-est de la France présentent peu d'originalité. Leur stabilité n'est remise en question qu'en situation de crise liée à un événement particulier ou à une intervention localisée, d'origine naturelle ou plus fréquemment anthropique. Ces crises déterminent une modification de l'équilibre hydrodynamique, qui peut se propager à des tronçons étendus de rivière et modifier le fonctionnement sur plusieurs décennies.

Cependant, les contrastes et écarts constatés entre les cours d'eau ou les tronçons de cours d'eau sont étroitement associés à la pente longitudinale des lits et des lignes d'eau. Si l'étape suivante de notre analyse doit mener à une quantification de ces écarts, en particulier par le calcul de paramètres hydrauliques, la détermination précise des pentes peut constituer un frein aux investigations. En effet, si les contrastes spatiaux de pente peuvent être mesurés, il est toujours difficile de prendre en considération les 
contrastes temporels, en particulier au moment des épisodes de crues, responsables du modelage des lits et de l'élaboration des indicateurs de l'évolution. Il faut donc trouver et définir des intervalles de confiance pertinents afin d'exprimer avec la meilleure précision possible, les raisons de l'évolution actuelle.

Néanmoins, les résultats déjà produits devraient contribuer dès maintenant à mieux comprendre la configuration des lits pour mieux choisir et mettre en oeuvre les actions de gestion, de protection ou encore de restauration des hydrosystèmes.

\section{BIBLIOGRAPHIE}

Agences de l'Eau. (1998) - SEQ physique : système d'évaluation de la qualité physique des cours d'eau, rapport de présentation. Rapport Inter-Agences, Ministère de l'Environnement, 31 p.

Amoros C., Petts G.E., 1993, Hydrosystèmes fluviaux, Masson Paris

Beck T., Corbonnois J., 2003, « Secteurs homogènes sur la Moselle amont ; méthode de découpage », Mosella, tome 27, n 1-2, février 2003

Beck T., Corbonnois J., 2003, « Les différents états du lit mineur de la Moselle entre Epinal et Flavigny, vers la reconstitution d'un nouvel équilibre dynamique », Actes du colloque Le fil de l'Eau, Nancy 17

Bernot V., Malavoi J.R., 1999, Méthode de sectorisation préalable à l'étude des cours d'eau. Guide d'utilisation pour l'application au Bassin Rhône-Méditerranée-Corse, ARALEPBP, Agence de l'Eau Rhône-Méditerranée-Corse, 29 p. + annexes

Bravard J.P., 2002, «Les réponses des systèmes fluviaux à une réduction des flux d'eau et de sédiments sous l'effet du reboisement en montagne », La Houille Blanche, 3, pp. 68-71

Corbonnois J., Humbert J., 2000, « Ressources et gestion de l'eau dans le nord-est de la France », in Bravard J.P, L'eau dans les régions françaises, d'un extrême à l'autre. SEDE, pp 219-249

Corbonnois J., Beck T., 2001, Cartographie de la dynamique fluviale de la Meurthe, Rapport CEGUM/ DIREN Lorraine, 65p. + annexe cartographique.

Corbonnois J., Beck T., 2002, Cartographie de la dynamique fluviale de la Moselle en amont du confluent de la Meurthe, Rapport CEGUM/DIREN Lorraine, 67p + annexe cartographique.

Corbonnois J., Beck T., Giovannacci L., 2004, « La dynamique fluviale des rivières lorraines d'origine vosgienne. Exemple de la Meurthe à Saint Clément ». Actes du colloque Spatialisation et cartographie en hydrologie, Metz, Mosella

Corbonnois J., Beck T., Jacquemot T., Bigarel R., 2003, Cartographie de la dynamique fluviale de la de la Vezouze et de la Mortagne, Rapport CEGUM/DIREN Lorraine, 59 p + annexe cartographique

Corbonnois J., Beck T., Maire G., Giovannacci L., 2003, « Subsiste-t-il encore sur la Meurthe, des tronçons de rivière à tracé naturel », in Ouvrage en hommage à R. Lambert, Université de Toulouse le Mirail 
Dawson F.H., Hornby D.D., Hilton J., 2002, A method for the automated extraction of environmental variables to help the classification of rivers in Britain. Aquatic Conservation : Marine and Freshwater Ecosystems 12, pp. 391-403.

Jacquemot T., Corbonnois J., De Ruffray S., 2004, « Hiérarchisation des processus de la dynamique fluviale par le traitement statistique des données », Actes du colloque Spatialisation et cartographie en hydrologie, Metz, Mosella

Kern K., 1992, "Restoration of lowland rivers: the german experience”, in Carling P.A. et Petts G.E. (Eds), Lowland floodplain rivers. Geomorphological perpectives, John Wiley \& Sons, pp. 279-297.

Liebault F.,Piégay H., 2001, “Assessment of channel changes due to long-term bedload supply decrease, Roubion River, France", Geomorphology, 36, pp. 167-186

Montgomery D.R., Buffington J.M., 1998, “Channel processes, classification, and response”, in Naiman R.J. et Bilby R.E. (Eds), River ecology and management, Lessons from the Pacific Coastal Ecoregion, Springer, pp. 13-42, 18

Schmitt L., Maire G., Humbert J., 2000, « Typologie hydro-géomorphologique des cours d'eau : vers un modèle adapté à la gestion du milieu physique des rivières du versant sud-occidental du fossé rhénan », Revue de Géographie de Lyon, 75, 4, pp. 347-363

Schmitt L., Maire G., Humbert J., 2001, « La puissance fluviale : définition, intérêt et limites pour une typologie hydro-géomorphologique de rivières ", Zeitschrift für Geomorphologie, 45, 2, pp.

$201-224$

\section{RÉSUMÉS}

L'évolution actuelle des lits fluviaux est largement influencée par les interventions humaines qui exacerbent la morphogenèse. Dans le Nord-Est de la France, en raison de pentes longitudinales faibles et de connexions lit mineur/lit majeur souvent préservées, les rivières ne réagissent que ponctuellement ou sur un temps assez long à ces interventions. Sapement des berges, déplacement latéral ou vertical du chenal sont beaucoup moins répandus (entre 20 et $40 \%$ de la longueur des rivières) que les états de stabilité, marqués par des berges fixées par la végétation (plus de 50\% de la longueur). Des indicateurs du fonctionnement géomorphologique sont définis à partir du traitement statistique de données qui décrivent l'état des lits, (cartographie sous SIG), de mesures (profils en travers) et d'une analyse de l'évolution sur quelques décennies. Les résultats doivent permettre de préciser les modes d'évolution et la vitesse de modification des lits fluviaux selon la nature (recoupement de sinuosité, extraction d'alluvions, fixation par enrochement des berges....) et le moment des actions anthropiques. Un schéma général de fonctionnement peut ainsi être proposé pour des cours d'eau du pourtour vosgien et du plateau lorrain. Mais dans ce dispositif, la Moselle occupe une place spécifique.

The current development of river beds is widely influenced by human intervention which exacerbates morphogenesis. In north-east France, because of weak gradients and connections between smaller and larger river beds which are often protected, rivers tend to react only occasionally, or rather over a long period of time to these interventions. Undermining of riverbanks and lateral or vertical displacement of the channel are much less widespread 2 (between $20 \%$ and $40 \%$ of the length of the rivers) than areas of stability, which are marked by those riverbanks that are strengthened with vegetation (more than $50 \%$ of the length). Indicators of geomorphological functioning are defined by statistical treatment of both the data that describes the state of the riverbeds (cartography under SIG), of the measurements taken, and 
also an analysis of their evolution over several decades. The results must allow one to specify the types of evolution occurring and the speed of modification of the river beds, as affected by nature (winding of meanders, transportation of alluvium, strengthening of banks by rock deposits) and also by anthropological actions. A general outline of functioning can thus be proposed for water courses at the perimeter of the Vosges and the plateau of the Lorraine. But within this plan, the Moselle takes a specific place.

\section{INDEX}

Keywords : fluvial morphology, Lorraine, river beds, river management

Mots-clés : aménagement, cours d'eau, dynamique fluviale, gestion, Lorraine

\section{AUTEURS}

\section{JEANNINE CORBONNOIS}

Université du Maine, UFR Lettres, Langues et Sc. Humaines, Avenue Olivier Messiaen, 72085 Le Mans Cedex 9, Tél : 02.43.82.76.97, jeannine.corbonnois@univ-lemans.fr

\section{THOMAS JACQUEMOT}

Cartographe, Bureau d'étude Inddigo, 9 rue Paulin Talabot, 31100 Toulouse, T.JACQUEMOT@inddigo.com

\section{LORIS GIOVANNACCI}

Chargé de mission au Parc naturel régional des Ballons des Vosges, 1 cour de l'Abbaye, 68140 Munster, l.giovannacci@parc-ballons-vosges.fr

\section{THIERRY BECK}

Université Paul Verlaine-METZ, UFR Sciences Humaines et Arts, Ile du Saulcy, BP 30309, 57006 METZ Cedex 1

\section{MOHAMED EL GHACH}

Université Sultan Moulay Slimane - Beni Mellal, Département de géographie, Équipe de Recherche "DPRP", Faculté des Lettres et des Sciences Humaines, Beni Mellal, elghachi_mohamed@yahoo.fr 